\title{
Multiplicity results for the two-vortex Chern-Simons Higgs model on the two-sphere
}

Weiyue Ding, Jürgen Jost, Jiayu Li and Guofang Wang

\begin{abstract}
We consider a Ginzburg-Landau type functional on $S^{2}$ with a $6^{\text {th }}$ order potential and the corresponding selfduality equations. We study the limiting behavior in the two vortex case when a coupling parameter tends to zero. This two vortex case is a limiting case for the Moser inequality, and we correspondingly detect a rich and varied asymptotic behavior depending on the position of the vortices. We exploit analogies with the Nirenberg problem for the prescribed Gauss curvature equation on $S^{2}$.
\end{abstract}

Keywords. Ginzburg-Landau functional, $\phi^{6}$ theory, Moser-Trudinger inequality, Nirenberg problem, phase transition, Chern-Simons Higgs theory.

\section{Introduction}

Functionals that exhibit a selfduality phenomenon in the sense that the absolute minimizers satisfy a set of first order partial differential equations are important in various areas of geometry and physics.

In the present paper, we investigate a special class of such functionals, namely Ginzburg-Landau type functionals with a $6^{\text {th }}$ order potential. Such functionals arise in Chern-Simons Higgs theories, as will be explained in $\S 2$. We consider a line bundle $L$ over a compact Riemann surface $\Sigma$, and the Lagrangian density

$$
\mathcal{L}(A, \phi)=\left|\nabla_{A} \phi\right|^{2}+\frac{k^{2}}{4} \frac{|F|^{2}}{|\phi|^{2}}+\frac{1}{k^{2}}|\phi|^{2}\left(1-|\phi|^{2}\right)^{2} .
$$

Here, $\phi$ is a section of $L$, and $A$ is a unitary connection on $L$ with curvature $F$. $k$ is a coupling parameter, and we are particularly interested in the limit analysis as $k$ tends to 0 . This limit analysis reveals a geometrically interesting phase transition that may also be relevant in superconductivity. The selfduality becomes manifest by rewriting

$$
L(A, \phi)=\int_{\Sigma} \mathcal{L}(A, \phi)=\int_{\Sigma}\left\{\left|\bar{\partial}_{A} \phi\right|^{2}+\left(\frac{k}{|\phi|} F+\frac{2}{k}|\phi|\left(|\phi|^{2}-1\right)\right)^{2}\right\}+\int_{\Sigma} F .
$$


If $\Sigma$ is compact, or, more generally, if one requires certain decay conditions at infinity,

$$
\int_{\Sigma} F=2 \pi N
$$

is a topologically quantity, where $N$ is an integer, the so-called vortex number that fixes the number of zeroes of $\phi$, the vortices. For $N \geq 0$, absolute minimizers of $L$ then have to satisfy the selfduality equations

$$
\begin{aligned}
\bar{\partial}_{A} \phi & =0 \\
F & =\frac{2}{k^{2}}|\phi|^{2}\left(1-|\phi|^{2}\right) .
\end{aligned}
$$

As $k \rightarrow 0$, one expects that the minima of the potential

$$
V(\phi)=|\phi|^{2}\left(1-|\phi|^{2}\right)^{2}
$$

at $|\phi|=1$ and $\phi=0$ dominate the behavior of minimizers of $L$, except that the topological constraint

$$
\int_{\Sigma} F=2 \pi N
$$

fixes the number of zeroes of $\phi$ as well as the integral of $F$. One thus expects a solution $\phi$ with $|\phi|$ close to one except in the vicinity of $N$ vortices. In the case were $\Sigma$ is a torus, such a solution has been constructed by Caffarelli-Yang $[\mathrm{CaY}]$. One also expects a solution that approaches 0 . Such a solution was recently obtained in an interesting paper of Tarantello [T] in case $N=1$, again for a torus. While the methods employed in the proofs of those results extend to the case of an arbitrary compact Riemann surface $\Sigma$, the method of Tarantello only works for $N=1$, because it depends on the Moser inequality. (She does obtain a second solution for arbitrary $N$, but as we shall see in the present paper, the limiting behavior will depend on $N$ in general.) Here we consider the case $N=2$ on the sphere $S^{2}$. This case is a limiting case for the Moser inequality, and consequently the analysis and the results become more subtle than for $N=1$. In fact, one may rewrite the selfduality equations by putting

$$
u(x)=\log |\phi(x)|^{2}
$$

to obtain

$$
\Delta u=\frac{4}{k^{2}} e^{u}\left(e^{u}-1\right)+4 \pi \sum_{j=1}^{N} \delta_{p_{j}}
$$

where $\delta_{p}$ is the Dirac distribution concentrated at $p$, and $p_{1}, \ldots, p_{N}$ are the prescribed zeroes of $\phi$, not necessarily all distinct. As will be explained in section 3 , 
in our case $N=2$ this equation can be related to the prescribed Gauss curvature equation

$$
\Delta u=-2 K e^{u}+2
$$

Thus, one expects that the methods developed for the Nirenberg problem, i.e. the existence problem for that equation, become relevant (see section3 for references). That is indeed the case, and in the present paper we shall obtain two families of solutions depending on the coupling parameter $k$ with a precise asymptotic behavior different from the one of the Caffarelli-Yang solution. The only exception is the case of a single vortex with multiplicity two where the Kazdan-Warner equation prevents the existence of a solution of the limiting equation and where we only find one additional family. Such a case distinction is not untypical for limit cases of embedding theorems. On the other hand, if the two vortices are antipodal, then an easy symmetry argument produces one-parameter solution spaces, i.e. infinitely many solutions for each sufficiently small value of $k$. The case of the torus has been investigated in our companion paper [DJLW] and by Nolasco-Tarantello $[\mathrm{NT}]$. By their results, it may be possible that a solution with a blow-up of the curvature at a non-vortex point also exists for certain conformal classes of tori.

In conclusion, the asymptotic analysis of the Chern-Simons Higgs functional considered here is much richer than the corresponding one for the GinzburgLandau functional with a $4^{\text {th }}$ order potential $\left(|\phi|^{2}-1\right)^{2}$. There, it was shown in [HJS] that asymptotically, as $k$ tends to $0, \phi$ becomes a covariantly constant section of $L$ with $|\phi|=1$, and the connection $A$ becomes flat, except near the vortices where all the topology concentrates. Solutions of the type found by Tarantello and in the present paper do not occur in that model. This is somewhat similar to the situation in the Seiberg-Witten functional that again has a $4^{\text {th }}$ order nonlinearity where the limiting analysis was carried out by Taubes [T3]. We expect that a Seiberg-Witten type functional with a $6^{\text {th }}$ order potential will exhibit very interesting features, partly analogous to the ones found in the present paper. We hope to be able to study this more closely. In fact, we consider the present analysis as a model study for that problem.

\section{The Chern-Simons Higgs model}

Let $S^{2}$ be the standard sphere in $\mathbb{R}^{3}$ with the standard metric $g_{0}$, and $M=\mathbb{R} \times S^{2}$ with the Lorentzian metric $g=d x_{0}^{2}-g_{0}$. Consider the (trivial) principal bundle $M \times U(1) \rightarrow M$. Let $A=-i A_{\mu} d x^{\mu}, A_{\mu}(x) \in \mathbb{R}, x=\left(x_{0}, x_{1}, x_{2}\right) \in M$ be a connection on this principal bundle. The curvature of $A$ is given by

$$
F_{A}=\frac{-i}{2} F_{\alpha, \beta} d x^{\alpha} \wedge d x^{\beta}
$$

with $F_{\alpha, \beta}=\partial_{\alpha} A_{\beta}-\partial_{\beta} A_{\alpha}, \quad \alpha, \beta=0,1,2$. The vector bundle associated to $M \times U(1)$ is $M \times \mathbb{C}$, where $\mathbb{C}$ is the complex plane. Let $\phi(x)$ be a section of the 
vector bundle $M \times \mathbb{C}$, i.e. $\phi(x)$ is a Higgs field, in physical notation. Let $D_{A} \phi$ denote $D_{\eta} \phi d x^{\eta}$ with $D_{\eta} \phi=\partial_{\eta} \phi-i A_{\eta} \phi$.

In this paper, we are interested in the following Chern-Simons-Higgs Lagrangian action density

$$
\mathcal{L}(A, \phi)=D_{\eta} \phi \overline{D_{\eta} \phi}+\frac{1}{4} k \varepsilon^{\alpha \beta \gamma} F_{\alpha \beta} A_{\gamma}-V(\phi)
$$

where $k>0$ is the coupling constant which determines the strength of the ChernSimons term $\varepsilon^{\alpha \beta \gamma} F_{\alpha \beta} A_{\gamma}, V(\phi)$ is the potential and the Levi-Civita tensor $\varepsilon^{\alpha \beta \gamma}$, $\alpha, \beta, \gamma=0,1,2$ is fixed by $\varepsilon^{012}=1$. This Lagrange density was first introduced by Hong-Kim-Pac in [HKP] and Jackiw-Weinberg in [JW].

The Euler-Lagrange equations for (2.1) are

$$
\left\{\begin{aligned}
\frac{1}{2} k \varepsilon^{\alpha \beta \gamma} F_{\alpha \beta} & =j^{\gamma}=i\left(\phi \overline{D^{\gamma} \phi}-\bar{\phi} D^{\gamma} \phi\right), \\
\Delta \phi & =-\frac{\partial V(\phi)}{\partial \phi}
\end{aligned}\right.
$$

where $j^{\gamma}$ is the conserved matter current density. We are interested in static solutions of $(2.2)$ with $V(\phi)=\frac{1}{k^{2}}|\phi|^{2}\left(1-|\phi|^{2}\right)$.

The energy density corresponding to the Lagrange density (2.1) is

$$
\mathcal{E}=\left|D_{0} \phi\right|^{2}+\left|D_{1} \phi\right|^{2}+\left|D_{2} \phi\right|^{2}+\frac{1}{k^{2}}|\phi|^{2}\left(1-|\phi|^{2}\right)
$$

supplemented by the Gauss law

$$
F_{12}=\frac{2}{k} J^{0}=-\frac{2 i}{k}\left(\phi \overline{D^{0} \phi}-\bar{\phi} D^{0} \phi\right) .
$$

Let $\bar{\partial}_{A} \phi=D_{1} \phi+i D_{2} \phi$. We have

$$
\left|D_{1} \phi\right|^{2}+\left|D_{2} \phi\right|^{2}=\left|\bar{\partial}_{A} \phi\right|^{2}+F_{12}|\phi|^{2}-\frac{1}{2} \varepsilon^{i k} \partial_{i} j_{k},
$$

Therefore, the energy density (2.3) may be written as

$$
\mathcal{E}=\frac{1}{4}\left(\frac{k}{|\phi|} F_{12}+\frac{2}{k}|\phi|\left(|\phi|^{2}-1\right)\right)^{2}+|\bar{\partial} \phi|^{2}+F_{12}+\operatorname{Im}\left\{\partial_{j} \varepsilon_{j k} \bar{\phi} D_{k} \phi\right\}
$$

where $\varepsilon_{j k}=-\varepsilon_{k j}, j, k=1,2$ and $\varepsilon_{12}=1$. Thus we obtain the following energy functional

$$
E(A, \phi)=\int_{S^{2}} \mathcal{E}=\int_{S^{2}} \frac{1}{4}\left|\frac{k}{|\phi|} F_{12}+\frac{2}{k}\right| \phi\left|\left(|\phi|^{2}-1\right)\right|^{2}+\int_{S^{2}}\left|\bar{\partial}_{A} \phi\right|^{2}+\int_{S^{2}} F_{12}
$$


The absolute minimizers of $E$ under the homotopically invariant constraint

$$
\frac{1}{2 \pi} \int_{S^{2}} F_{12}=N
$$

satisfy the Bogomolny type self-dual equations

$$
\begin{cases}\bar{\partial}_{A} \phi & =0, \\ F_{12}+\frac{2}{k^{2}}|\phi|^{2}\left(|\phi|^{2}-1\right) & =0,\end{cases}
$$

with the Gauss law $k F_{12}+2 A_{0}|\phi|^{2}=0$ (see (2.4)). Here $N$ is an integer. One can easily check that a solution of (2.7) with the Gauss law satisfies (2.2). In this paper, we are interested in finding such special solutions of (2.2).

As in $[\mathrm{CaY}]$ and $[\mathrm{T}]$, one can first obtain a maximal solution as follows

Theorem 2.1. ([CaY]). Let $p_{1}, \ldots, p_{m}$ be given points (or vortices) on $S^{2}$ and $n_{1}, \ldots, n_{m}$ positive integers such that $\sum_{j=1}^{m} n_{j}=N \geq 0$. There exists a $k_{c} \in$ $\left(0, \frac{1}{2} \sqrt{\left|S^{2}\right| / \pi N}\right)$ such that (2.7) admits a solution $\left(A_{k}, \phi_{k}\right)$ for which $p_{1}, \ldots, p_{m}$ are the zeroes of $\phi$ with multiplicity $n_{1}, \ldots n_{m}$ if and only if $0<k \leq k_{c}$. Moreover

(i) The energy, magnetic flux and electric change of $\left(A_{k}, \phi_{k}\right)$ are respectively given by

$$
E=2 \pi N, \Phi=2 \pi N, Q=2 \pi k N
$$

(ii) The solution $\left(A_{k}, \phi_{k}\right)$ is maximal in the sense that if $\left(A^{\prime}, \phi^{\prime}\right)$ is another solution of (2.7) with the same vortices as $(A, \phi)$, then $\left|\phi^{\prime}\right|<|\phi|$.

(iii) $\left|\phi_{k}\right|<1$ in $S^{2}$ and $\left|\phi_{k}\right| \rightarrow 1$ as $k \rightarrow 0$ a.e. in $S^{2}$ and in $H^{1, q}\left(S^{2}\right), 1<$ $q<2$.

$$
F_{12}^{(k)} \rightarrow 2 \pi \sum_{j=1}^{N} \delta_{p_{j}} \text { in the sense of measures as } k \rightarrow 0 .
$$

where each Dirac distribution $\delta_{p_{j}}$ occurs with multiplicity $n_{j}, j=1, \ldots, m$.

One can also obtain another solution by using the mountain pass Lemma as [T]. Here we are interested in solutions of (2.7) with a different asymptotic behavior when $k \rightarrow 0$. Motivated by Caffarelli-Yang's variational method, when $N=1$, Tarantello obtained in $[\mathrm{T}]$,

Theorem 2.2. There exists a solution $\left(\tilde{A}_{k}, \tilde{\phi}_{k}\right)$ of (2.7) for small $k>0$ such that (2.8) holds and $\left\|\tilde{\phi}_{k}\right\|_{C^{q}\left(S^{2}\right)} \rightarrow 0$ as $k \rightarrow 0$ for any $q \geq 0$.

Although they did not consider (2.7) on $S^{2}$, the methods of Caffarelli-Yang and Tarantello extend to this case.

Tarantello used the Moser inequality [M1,2] to study this problem. Here we consider the case $N=2$. As we already mentioned in the introduction, this case 
is a limiting case (a critical case). The main difficulty to find solutions of (2.7) is the lack of a coercivity condition. A crucial observation is that our problem can be seen as a perturbation of the well-known Nirenberg problem. Hence, methods developed in the Nirenberg problem may be used to study (2.7). First we obtain a solution of (2.7) which has a new asymptotic behavior as $k \rightarrow 0$.

Theorem 2.3. Let $N=2$ and $P_{ \pm}$two vortices. For small $k>0$, there exists a solution $\left(A_{k}^{2}, \phi_{k}^{2}\right)$ such that

(i) (2.8) holds,

(ii) $\left|\phi_{k}^{2}\right| \rightarrow 0$ in $C^{0}$ uniformly,

(iii) $F_{12}\left(A_{k}^{2}\right) \rightarrow 4 \pi \delta_{Q}$,

where $Q \neq P_{ \pm}$is determined by $P_{ \pm}$(see section 3.). Moreover, if $P_{+}=-P_{-}$there exists a family of solutions $\left(A_{k}^{2}(\vartheta), \phi_{k}^{2}(\vartheta)\right)$ such that (i), (ii) and (iii) hold with $T_{\vartheta} Q$, where $T_{\vartheta}$ is the rotation with angle $\vartheta$ about the axis from $P_{+}$to $P_{-}$.

This is a new interesting situation. We guess that such a solution exists in the general case.

Theorem 2.4. Let $N=2$ and $P_{ \pm}$two vortices. If $P_{+} \neq P_{-}$, then for small $k$ there exists another solution $\left(A_{k}^{3}, \phi_{k}^{3}\right)$ of (2.7) such that

(i) (2.8) holds,

(ii) $\phi_{k}^{3} \rightarrow 0$ in $C^{q}$, as $k \rightarrow 0$, for any $q \geq 0$.

The potential $|\phi|^{2}\left(|\phi|^{2}-1\right)^{2}$ has a minimum at $|\phi|=1$ and at $\phi=0$. The solution of Theorem 2.1 corresponds to the minimum at $|\phi|=1$, the one of Theorem 2.3 to the one at $\phi=0$, while the solution of Theorem 2.4 is a saddle point solution for an associated functional. Of course, the vortices prevent that $|\phi|=1$ or $\phi \equiv 0$ are exact solutions, but in the limit $k \rightarrow 0$, the obstructions concentrate at isolated points. According to the theorems, for $N=2$, we have 3 different cases for small $k$.

(1) If $P_{+}=P_{-},(2.7)$ admits at least two solutions,

(2) If $P_{+}=-P_{-},(2.7)$ admits infinitely many solutions,

(3) If $P_{+} \neq \pm P_{-},(2.7)$ admits at least three solutions.

It is clear that case (3) is the generic case. Before we start to prove the theorems, we first reduce (2.7) to a semilinear equation. Such a reduction was first used by Taubes in [T1], [T2].

It is clear that the first equation of (2.7) may be written as

$$
2 \bar{\partial} \phi-i A \phi=0
$$

where $A=A_{1}+i A_{2}$ and $\bar{\partial}=\frac{1}{2}\left(\partial_{1}+i \partial_{2}\right)$ is the usual Cauchy-Riemann operator. Hence $\phi$ can be considered as a holomorphic section of a line bundle, and it there- 
fore admits a finite number of zeroes in $S^{2}$ with integer multiplicities. Outside the zero set of $\phi, Z(\phi)$, we have

$$
A=-2 i \bar{\partial} \log \phi
$$

Set $u(x)=\log |\phi(x)|^{2}$. From (2.7) and (2.11) $u$ satisfies

$$
\Delta u=\frac{4}{k^{2}} e^{u}\left(e^{u}-1\right) \text { in } S^{2} \backslash Z(\phi)
$$

and

$$
u(z)=n_{k} \log \left|z-P_{k}\right|^{2} \text { as } z \rightarrow P_{k} .
$$

On the other hand, if we have a solution $u$ of (2.12)-(2.13), set

$$
\phi(z)=\exp \left(\frac{1}{2} u(z)+i \sum_{j=1}^{N} \arg \left(z-P_{j}\right)\right)
$$

and $A=-2 i \bar{\partial} \log \phi$, then one can check that $(A, \phi)$ satisfies (2.7). Therefore, we only have to consider (2.12) and (2.13). Clearly (2.12)-(2.13) is equivalent to

$$
\Delta u=\frac{4}{k^{2}} e^{u}\left(e^{u}-1\right)+4 \pi \sum_{j=1}^{N} \delta_{P_{j}}
$$

where $\delta_{P}$ is the Dirac distribution concentrated at $P$.

\section{Proof of Theorem 2.3}

Let $P_{+}$and $P_{-}$be two vortices on $S^{2}$. Let $u_{0}$ be the unique solution of

$$
\left\{\begin{array}{l}
\Delta u_{0}=-2+4 \pi\left(\delta_{P_{+}}+\delta_{P_{-}}\right), \text {in } S^{2} \\
\int_{S^{2}} u_{0}=0 .
\end{array}\right.
$$

Let $\lambda=4 / k^{2}$ and $K=e^{u_{0}}$. (2.12) is equivalent to

$$
\Delta u=\lambda K e^{u}\left(K e^{u}-1\right)+2 \text { in } S^{2} .
$$

We first summarize some simple properties of $K$ in three different cases.

Lemma 3.1. case (i): $P+=-P_{-}$. After a change of coordinates, we may assume that $P_{+}$is the north pole. Then $K$ is axially symmetric, i.e. invariant under rotations about the axis between north and south pole, i.e. between $P_{+}$and $P_{-}$, as well as invariant under reflections about the equator of $S^{2}$, i.e. $K(x)=$ $K(-x)$ for all $x \in S^{2}$. $K$ achieves its maximum for any point on the equator. 
case (ii): $P_{+}=P_{-}$, i.e. $P_{+}$is a double vortex. $K$ is again axially symmetric about the line between $P_{+}$and $-P_{+}$. It achieves its unique maximum at $-P_{+}$.

case (iii): $P_{+} \neq \pm P_{-}$(the generic case). $K$ has a unique maximum point $\bar{Q} \neq P_{+}, P_{-}$and a unique saddle point $Q=-\bar{Q}$.

Equation (3.2) is the Euler-Lagrange equation of the following functional

$$
I_{\lambda}(u)=f \frac{1}{2}|\nabla u|^{2}+2 u+\frac{\lambda}{2}\left(K e^{u}-1\right)^{2},
$$

where $f u$ is the average of $u$ over $S^{2}$, i.e. $f u=\frac{1}{4 \pi} \int_{S^{2}} u$. As in [T], motivated by the variational method used in $[\mathrm{CaY}]$, we consider the following functional

$$
\begin{aligned}
J_{\lambda}(u) & =f\left(\frac{1}{2}|\nabla u|^{2}+2 u+\frac{\lambda}{2}\left(K e^{(u+\rho(u))}-1\right)^{2}\right) \\
& +2 \rho(u)-\frac{\lambda}{2}+2 \log \lambda
\end{aligned}
$$

in

$$
\mathcal{A}_{\lambda}=\left\{u \in H^{1,2}\left(S^{2}\right) \mid f e^{u}=1 \&\left(f K e^{u}\right)^{2}-\frac{8}{\lambda} f K^{2} e^{2 u} \geq 0\right\},
$$

where

$$
\rho(u)=\log \left(\frac{f K e^{u}-\sqrt{\left(f K e^{u}\right)^{2}-\frac{8}{\lambda} f K^{2} e^{2 u}}}{f K^{2} e^{2 u}}\right) .
$$

The term $-\frac{\lambda}{2}+\log \lambda$ ensures that $J_{\lambda}$ has a uniform lower bound (see Lemma 3.4 below). This value of $\rho(u)$ is needed to satisfy the constraint that comes from integrating (3.2). Alternatively, this value of $\rho(u)$ is determined by minimizing $J_{\lambda}$ among functions of the form $u+\rho$ w.r.t. $\rho$ for given $u$ satisfying $f e^{u}=1$.

Set $H=\left\{u \in H^{1,2}\left(S^{2}\right) \mid f e^{u}=1\right\}$.

Lemma 3.2. If $u \in \stackrel{\circ}{\mathcal{A}}_{\lambda}$, the interior of $\mathcal{A}_{\lambda}$, is a critical point of $J_{\lambda}$, then $v=$ $u+\rho(u)$ is a solution of (3.2).

Proof. The proof is straightforward (cf. [T]).

A crucial observation is that we may rewrite $J_{\lambda}$ in a suitable form as follows. By the definition of $\rho$, we have

$$
\lambda e^{\rho(u)} f K e^{u}=\frac{4 f K e^{u}}{f K e^{u}+\sqrt{\left(f K e^{u}\right)^{2}-\frac{8}{\lambda} f K^{2} e^{2 u}}} .
$$


Consequently,

$$
2<\lambda e^{\rho(u)} f K e^{u} \leq 4, \text { for any } u \in \mathcal{A}_{\lambda} .
$$

Set $B_{\lambda}(u)=\lambda e^{\rho(u)} f K e^{u}$. Again, by definition, we have

$$
\lambda f K^{2} e^{2(u+\rho(u))}-\lambda f K e^{u+\rho(u)}=-2 .
$$

Thus, we can rewrite $J_{\lambda}$ as follows (deleting an irrelevant additive constant)

$$
J_{\lambda}(u)=f\left(\frac{1}{2}|\nabla u|^{2}+2 u\right)-2 \log f K e^{u}+2 \log B_{\lambda}(u)-\frac{1}{2} B_{\lambda}(u)
$$

Set $\alpha_{\lambda}=\inf _{u \in \mathcal{A}_{\lambda}} J_{\lambda}(u)$. In this section, we shall prove that $\alpha_{\lambda}$ is achieved by some $\underline{u}_{\lambda} \in \stackrel{\circ}{\mathcal{A}}_{\lambda}$. For simplicity of notation, let $f(t)=2 \log t-\frac{1}{2} t$ and $f_{\lambda}=f \circ B_{\lambda}$. Then $J_{\lambda}$ is written as

$$
J_{\lambda}=J+f_{\lambda}
$$

where

$$
J(u)=f\left(\frac{1}{2}|\nabla u|^{2}+2 u\right)-2 \log f K e^{u} .
$$

The corresponding Euler-Lagrange equation of $J$ is given by

$$
\Delta u=-2 K e^{u}+2
$$

which is the so-called prescribed Gauss curvature equation. The corresponding problem of existence of solutions of (3.7) is called the Nirenberg problem. This problem has been studied by many mathematicians. (See $[\mathrm{M} 2],[\mathrm{A}],[\mathrm{H}],[\mathrm{CD}]$, $[\mathrm{CY} 1,2]$ and $[\mathrm{CkL}]$ and references therein.) $J_{\lambda}$ can be considered as a perturbation of $J$ for large $\lambda$. So it is natural to apply methods developed for the Nirenberg problem in our problem.

Now let us first introduce the definition of the center of mass of a function $u \in H^{1,2}\left(S^{2}\right)$ which was first used in [CD] in the Nirenberg problem. For $u \in$ $H^{1,2}\left(S^{2}\right)$, the center of mass is defined as

$$
P(u)=\frac{f_{S^{2}} x e^{u}}{f_{S^{2}} e^{u}} .
$$

Given $q \in S^{2}$, we choose coordinates $x=\left(x_{1}, x_{2}, x_{3}\right) \in S^{2}$ such that $q=(0,0,1)$. The stereographic projection $\Pi: S^{2} \rightarrow \hat{\mathbb{C}}=\mathbb{C} \cup\{\infty\}$ with respect to $q$ is defined by

$$
\left(x_{1}, x_{2}, x_{3}\right) \mapsto z=\frac{x_{1}+i x_{2}}{1-x_{3}} .
$$


For $t>0$, let $m_{t}: \mathbb{C} \rightarrow \mathbb{C}$ be the usual multiplication by $t$, i.e $m_{t}(z)=t z$ for any $z \in \mathbb{C}$. For any $u \in H^{1,2}\left(S^{2}\right)$, there is $(q, t) \in S^{2} \times[1,+\infty)$ and

$$
w=u_{\varphi_{q, t}}:=u \circ \varphi_{q, t}+\psi_{q, t} .
$$

such that $P(w)=0$, where $\varphi_{q, t}=\Pi^{-1} \circ m_{t} \circ \Pi$ and $\psi_{q, t}=\log \operatorname{det}\left(d \varphi_{q, t}\right)$. (Note that our notation differs slightly from the one in [CY1] and [CkL]). In [CkL], (see also $[\mathrm{O}],[\mathrm{CD}]$ and $[\mathrm{CY} 1])$ the authors proved

Lemma 3.3. $H$ is diffeomorphic to $H^{0} \times B^{3}$ by sending $u \in H$ to $\left(w=u_{\varphi_{q, t}}, q, 1-\right.$ $\left.t^{-2} \log t\right)$, where $H=\left\{u \in H^{1,2}\left(S^{2}\right): f e^{u}=1\right\}$ and $H^{0}=\{u \in H \mid P(u)=0\}$.

Now we can rewrite $J_{\lambda}$ by this decomposition. First, let $S(u)=\int \frac{1}{2}|\nabla u|^{2}+2 u$. It is important that $S$ is invariant under conformal transformations, namely,

$$
S(u)=S\left(u_{\varphi_{q, t}}\right)
$$

for any conformal transformations $\varphi_{q, t}$ of $S^{2}$. Let $u=(w, q, t) \in H$. We write $J_{\lambda}$ as

$$
J_{\lambda}=J_{\lambda}(w, q, t)=S(w)-2 \log f K \circ \varphi_{q, t} e^{w}+f_{\lambda}(w, q),
$$

where

$$
f_{\lambda}(u)=f \circ B_{\lambda}(w, q, t)
$$

and

$$
B_{\lambda}(w, q, t)=4\left(1+\sqrt{1-\frac{8}{\lambda} \frac{f K^{2} \circ \varphi_{q, t} e^{2 w}\left(\operatorname{det}\left(d \varphi_{q, t}\right)\right)^{-1}}{\left(f K \circ \varphi_{q, t} e^{w}\right)^{2}}}\right)^{-1}
$$

For simplicity of notation, let

$b(u)=b(w, q, t)=f K^{2} \circ \varphi_{q, t} e^{2 w}\left(\operatorname{det}\left(d \varphi_{q, t}\right)^{-1}\right) /\left(f K \circ \varphi_{q, t} e^{w}\right)^{2}$. We need

Lemma 3.4. ([CY1]). If $u \in H^{0}$, then $f|\nabla u|^{2} \leq 2\left(1-a_{0}\right)^{-1} S(w)$ for a constant $a_{0}<1$.

The following asymptotic behavior for large $t$ is crucial for the proofs of the Theorems.

Lemma 3.5. For any $b_{0}>0$, we have for all $w$ with $S(w) \leq b_{0}$ uniformly in $t$ as $t \rightarrow \infty$

(i) $f K \circ \varphi_{q, t} e^{w}=K(q)+O\left(t^{-1} \log 1 / 2 t\right)$ 
(ii)

$$
f K^{2} \circ \varphi_{q, t} e^{2 w}\left(\operatorname{det}\left(d \varphi_{q, t}\right)\right)^{-1}=t^{2} K^{2}(q) f e^{2 w}\left(\frac{1+x_{3}}{2}\right)^{2}+O(t),
$$

(iii)

$$
f K^{4} \circ \varphi_{q, t} e^{4 w}\left(\operatorname{det}\left(d \varphi_{q, t}\right)\right)^{-2}=t^{4} K^{4}(q) f e^{4 w}\left(\frac{1+x_{3}}{2}\right)^{4}+O\left(t^{3}\right) .
$$

Proof. (i) was proved in [CY1].

(ii) We use the plane coordinates induced from the stereographic projection with respect to $q$ (see above). By the Taylor expansion of $K$ around $q=(0,0,1)$, we have

$$
K(x)=K(q)+a_{1} x_{1}+a_{2} x_{2}+O(|x|)
$$

in $\{x \in \mathbb{C}|| x \mid \geq M\}$ for a fixed large $M>0$. By a direct computation, we have

$$
\operatorname{det}\left(d \varphi_{q, t}\right)(z)=t^{2}\left(\frac{1+|z|^{2}}{1+t^{2}|z|^{2}}\right)^{2}
$$

Let $R_{t}=\{z \in \mathbb{C}|| z \mid \geq M / t\}$ and $R_{t}^{c}=\mathbb{C} \backslash R_{t}$. We decompose the left hand side of (ii) as follows

$$
\frac{1}{4 \pi}\left(\int_{R_{t}}+\int_{R_{t}^{c}}\right) K^{2}(t z) e^{2 w(z)} t^{-2} \frac{\left(1+t^{2}|z|^{2}\right)^{2}}{\left(1+|z|^{2}\right)^{2}} d A(z)
$$

where $d A(z)=\frac{d|z|^{2}}{\left(1+|z|^{2}\right)^{2}}$. A direct computation shows that

$$
\int_{R_{t}^{c}} K^{2}(t z) e^{2 w(z)} t^{-2} \frac{\left(1+t^{2}|z|^{2}\right)^{2}}{\left(1+|z|^{2}\right)^{2}} d A(z) \leq O\left(t^{-2}\right)
$$

and

$$
\begin{gathered}
\int_{R_{t}} x_{1}(t z) e^{2 w(z)} t^{-2} \frac{\left(1+t^{2}|z|^{2}\right)^{2}}{\left(1+|z|^{2}\right)^{2}} d A(z) \\
\leq 2 \int_{R_{t}} \frac{t|z|}{1+t^{2}|z|^{2}} e^{2 w(z)} t^{-2} \frac{\left(1+t^{2}|z|^{2}\right)^{2}}{\left(1+|z|^{2}\right)^{2}} d A(z) \\
=2 \int_{R_{t}} \frac{1+t^{2}|z|^{2}}{1+|z|^{2}} t^{-1}|z| e^{2 w(z)} d A(z)
\end{gathered}
$$


Vol. 74 (1999)

$$
\begin{gathered}
=4 \int_{|z| \geq \frac{M}{t}} \frac{1+t^{2}|z|^{2}}{1+|z|^{2}} t^{-1}|z| e^{2 w(z)} \frac{|z|}{\left(1+|z|^{2}\right)^{2}} d|z| \\
\leq 8 t \int_{|z| \leq \frac{M}{t}} \frac{|z|^{4}}{\left(1+|z|^{2}\right)^{3}} d|z| \\
\leq O(t) .
\end{gathered}
$$

Similar

$$
\int_{R_{t}} x_{2}(t z) e^{2 w(z)} t^{-2} \frac{\left(1+t^{2}|z|^{2}\right)^{2}}{\left(1+|z|^{2}\right)^{2}} d A(z)=O(t)
$$

We also have

$$
\begin{aligned}
& \int_{R_{t}} K^{2}(t z) e^{2 w(z)} t^{-2} \frac{\left(1+t^{2}|z|^{2}\right)^{2}}{\left(1+|z|^{2}\right)^{2}} d A(z) \\
= & t^{2} \int_{R_{t}} K^{2}(q) e^{2 w(z)} \frac{|z|^{4}}{\left(1+|z|^{2}\right)^{2}} d A(z)+O(t) \\
= & t^{2} f K^{2}(q) e^{2 w(z)}\left(\frac{1+x_{3}}{2}\right)^{2} d A(z)+O(t) .
\end{aligned}
$$

for $z=\left(x_{1}+i x_{2}\right) /\left(1-x_{3}\right)$. The preceding estimates prove (ii). (iii) The proof is similar to that of (ii).

Lemma 3.6. ([H], $[\mathrm{CY} 2]) \operatorname{Inf}_{u \in H} J(u)=-\log \left(\max _{x \in S^{2}} K(x)\right)$, and $J$ does not achieve its infimum, if $K$ is not constant.

Now we have

Proposition 3.7. For all sufficiently large $\lambda$, there exists $\underline{u}_{\lambda} \in \stackrel{\circ}{\mathcal{A}}_{\lambda}$ with $J_{\lambda}\left(\underline{u}_{\lambda}\right)=$ $\alpha_{\lambda}$.

To prove the proposition, we need one more Lemma. We recall $\alpha_{\lambda}=\inf _{u \in \mathcal{A}_{\lambda}} J_{\lambda}(u)$.

Lemma 3.8. (i) $\lim _{\lambda \rightarrow+\infty} \alpha_{\lambda}=-\log (\max K)-1+2 \log 2$

(ii) $\inf _{u \in \partial \mathcal{A}_{\lambda}} J_{\lambda}(u) \geq-\log (\max K)-2+2 \log 4$.

Proof. By Lemma 3.6, for any $\varepsilon>0$ there exists $u_{0} \in H$ with

$$
J\left(u_{0}\right) \leq-\log (\max K)+\varepsilon / 2
$$

On the other hand, $\mathcal{B}_{\lambda}:=\left\{u \in \mathcal{A}_{\lambda} \mid b(u) \leq \log \lambda\right\}$ converges to $H$ as $\lambda \rightarrow+\infty$. Hence we can choose $\lambda_{0}$ such that $u_{0} \in \mathcal{B}_{\lambda}$ for any $\lambda \geq \lambda_{0}$. If we choose $\lambda_{0}$ large 
enough, we have

$$
\begin{aligned}
J_{\lambda}\left(u_{0}\right) & =J\left(u_{0}\right)+f_{\lambda}\left(u_{0}\right) \\
& \leq-\log (\max K)+\varepsilon / 2+f\left(\frac{1}{1+\sqrt{1-\frac{8}{\lambda} \log \lambda}}\right) \\
& \leq-\log (\max K)-1+2 \log 2+\varepsilon
\end{aligned}
$$

Clearly, for any $u \in \mathcal{A}_{\lambda}, J_{\lambda}(u) \geq-\log (\max K)-1+2 \log 2$. Hence $\lim _{\lambda \rightarrow \infty} \alpha_{\lambda}=$ $-\log (\max K)-1+2 \log 2$.

(ii) If $u \in \partial \mathcal{A}_{\lambda}$, by definition, $f \circ B_{\lambda}(u)=-2+2 \log 4$. Hence $J_{\lambda}(u) \geq-\log (\max K)-$ $2+2 \log 4$.

Remark. By Lemma 3.8, $\lim _{\lambda \rightarrow \infty} \alpha_{\lambda}<\inf _{u \in \partial \mathcal{A}_{\lambda}} J_{\lambda}(u)$ for any $\lambda$.

Proof of Proposition 3.7. Consider a minimizing sequence $\left\{u_{i}\right\} \in \mathcal{A}_{\lambda}$ of $J_{\lambda}$. According to Lemma 3.3 , we rewrite it as $u_{i}=\left(w_{i}, q_{i}, t_{i}\right)$. First we claim that $S\left(w_{i}\right)$ is bounded. This is easy to prove, for $f_{\lambda}\left(u_{i}\right)$ is bounded and $-\log f K \circ \varphi_{q_{i}, t_{i}} e^{u_{i}} \geq$ $-\log (\max K)$. By Lemma 3.4, the boundedness of $S\left(w_{i}\right)$ implies that

$$
f\left|\nabla w_{i}\right|^{2} \text { is bounded. }
$$

Second, we claim that $\left\{t_{i}\right\}$ is also bounded. Assume by contradiction that $\left\{t_{i}\right\}$ is unbounded. There are two possibilities

(1) $q_{i} \rightarrow q$ and $q$ is one of the vortices,

(2) $q_{i} \rightarrow q$ and $q$ is not equal to $P_{+}$or $P_{-}$.

Case (1). Since $S\left(w_{i}\right)$ is bounded, from (i) of Lemma 3.5 we have

$$
-\log f K \circ \varphi_{q_{i}, t_{i}} e^{w_{i}} \rightarrow+\infty
$$

as $i \rightarrow+\infty$. Since $S\left(w_{i}\right) \geq 0$ and $f_{\lambda}$ is bounded, it follows that $J_{\lambda}\left(u_{i}\right) \rightarrow+\infty$, a contradiction.

Case (2). Recalling (3.8), (3.9) and the boundedness of $f_{\lambda}\left(u_{i}\right)$, Lemma 3.5 implies that

$$
t_{i}^{2} f e^{2 w_{i}}\left(1+x_{3}\right)^{2} \leq c \lambda .
$$

Recall that we have

$$
t_{i}^{2} f e^{2 w_{i}}=1 \text { and } f e^{w_{i}} x_{3}=0 .
$$


This implies that

$$
f e^{2 w_{i}}\left(1+x_{3}\right)^{2} \geq f e^{w_{i}}\left(1+x_{3}\right)=1
$$

So

$$
t_{i}^{2} \leq c \lambda
$$

is bounded.

Now it is clear that the boundedness of $\left\{t_{i}\right\}$ implies that of $\left\{\left\|u_{i}\right\|_{H^{1,2}}\right\}$. Hence we may assume that there exists $\underline{u}_{\lambda} \in H^{1,2}\left(S^{2}\right)$ such that $u_{i}$ converges to $\underline{u}_{\lambda}$ weakly in $H^{1,2}$, and strongly in $L^{q}$ for any $q>1$ and almost everywhere in $S^{2}$. It follows that

$$
f K e^{\underline{u}_{i}} \rightarrow f K e^{\underline{u}_{\lambda}}
$$

and

$$
f K^{2} e^{2 \underline{u}_{i}} \rightarrow f K^{2} e^{2 \underline{u}_{\lambda}}
$$

as $i \rightarrow+\infty$. Therefore $J_{\lambda}\left(\underline{u}_{\lambda}\right) \leq \alpha_{\lambda}$ and $\underline{u}_{\lambda} \in \mathcal{A}_{\lambda}$. Now, in view of Lemma 3.8, $\underline{u}_{\lambda} \in \stackrel{\circ}{\mathcal{A}}_{\lambda}$.

Remark. We can prove the proposition in a different way which does not use conformal transformations. Actually, we can prove proposition 3.7 for any compact surface in [DJLW].

By Lemma 3.2, we know that $\underline{u}_{\lambda}+\rho\left(\underline{u}_{\lambda}\right)$ is a solution of (3.2).

Now we consider the behavior of $\underline{u}_{\lambda}$ as $\lambda \rightarrow+\infty$. First, it is clear that $\underline{u}_{\lambda}$ cannot converge in $H^{1,2}$. Otherwise, we can obtain a minimum of $J$ in $H$, which contradicts Lemma 3.6.

Proposition 3.9. If we write $\underline{u}_{\lambda}$ as $\left(w_{\lambda}, q_{\lambda}, t_{\lambda}\right)$ then $w_{\lambda} \rightarrow 0$ strongly in $H^{1,2}, t_{\lambda} \rightarrow$ $\infty$ and $q_{\lambda} \rightarrow Q$ as $\lambda \rightarrow+\infty$, where $Q$ is one of the maximum points of $K$. Moreover, $w_{\lambda} \rightarrow 0$ strongly in $C^{1}$ as $\lambda \rightarrow+\infty$.

Proof. Since $\lim _{\lambda \rightarrow \infty} \alpha_{\lambda}=-\log (\max K)-1+2 \log 2,\left\{S\left(w_{\lambda}\right)\right\}$, hence $\left\{\left\|w_{\lambda}\right\|_{H^{1,2}}\right\}$ is bounded. From the above discussion, we know that $\left\{t_{\lambda}\right\}$ is unbounded. Assume $q_{\lambda} \rightarrow Q$ and $w_{\lambda} \rightarrow w_{0}$ weakly in $H^{1,2}$ as $\lambda \rightarrow \infty$. By a direct computation we have

$$
\lim _{\lambda \rightarrow \infty} \alpha_{\lambda}=\lim _{\lambda \rightarrow \infty} J_{\lambda}\left(u_{\lambda}\right) \geq S\left(w_{0}\right)-\log K(Q)-1+2 \log 2
$$

Consequently, $S\left(w_{0}\right)=0$, hence $w_{0} \equiv 0 . \quad K(Q)=\max K$ and $w_{\lambda}$ converges to $w_{0}=0$ strongly in $H^{1,2}$. Clearly, $w_{\lambda}$ satisfies a suitable equation similar to (3.7), 
from which we can show that $w_{\lambda} \rightarrow 0$ strongly in $C^{1}$ as $\lambda \rightarrow+\infty$ by elliptic estimates.

Corollary 3.10. When $P_{+}=-P_{-}$, there are infinitely many solutions of (3.2).

Proof. In this case, $K$ is axially symmetric along the axis crossing $P_{+}$and $P_{-}$. Denote by $T_{\vartheta}$ the rotation along this axis with angle $\vartheta$. It is clear that $T_{\vartheta} \underline{u}_{\lambda}$ is also a critical point of $J_{\lambda}$ for any $\vartheta \in(0,2 \lambda]$. From the previous proposition, we know $P\left(\underline{u}_{\lambda}\right) \rightarrow Q$ as $\lambda \rightarrow \infty$. Hence, for large $\lambda, P\left(\underline{u}_{\lambda}\right)$ is not the origin of $\mathbb{R}^{3}$. On the other hand, it is clear that $T_{\vartheta}\left(P\left(\underline{u}_{\lambda}\right)\right)=P\left(T_{\vartheta} \underline{u}_{\lambda}\right)$ and $T_{\vartheta}$ has no fixed points except the origin. Hence $P\left(T_{\vartheta} \underline{u}_{\lambda}\right) \neq P\left(\underline{u}_{\lambda}\right)$ for any $\vartheta \in(0,2 \pi]$, which implies

$$
\underline{u}_{\lambda} \neq T_{\vartheta} u_{\lambda} \text { for any } \vartheta \in(0,2 \lambda] \text {. }
$$

Hence (3.2) admits infinitely many solutions.

Now we can prove Theorem 2.3.

Proof of Theorem 2.3. From Propositions 3.8 and 3.9, all properties except (ii) are easy to check. Now we prove (ii). Recall that $\left|\phi_{\lambda}\right|=e^{u_{0}+\underline{u}_{\lambda}+\rho\left(\underline{u}_{\lambda}\right)}=K e^{\underline{u}_{\lambda}+\rho\left(\underline{u}_{\lambda}\right)}$. We claim

$$
\frac{t_{\lambda}^{2}}{\lambda} \rightarrow 0 \quad \text { as } \lambda \rightarrow \infty
$$

where $\underline{u}_{\lambda}=\left(w_{\lambda}, q_{\lambda}, t_{\lambda}\right)$. If the claim is true, by (3.5) and Lemma 3.5 we have

$$
\left.\lambda e^{\rho\left(u_{\lambda}\right.}\right) \rightarrow \frac{2}{K(Q)} \quad \text { as } \lambda \rightarrow+\infty .
$$

By Proposition 3.9, we can show that

$$
\max e^{\underline{u}_{\lambda}} \leq c t_{\lambda}^{2}
$$

for some constant $c>0$. In fact, we have

$$
e^{\underline{u}_{\lambda} \circ \varphi_{q, t}}=e^{w_{\lambda}}\left(\operatorname{det} d \varphi_{q, t}\right)^{-1}
$$

and $w_{\lambda} \rightarrow 0$ strongly in $C^{1}$. Hence, we have

$$
\begin{aligned}
\left|\phi_{\lambda}\right| & =K e^{\underline{u}_{\lambda}+\rho\left(\underline{u}_{\lambda}\right)} \\
& \leq c \lambda^{-1} t^{2} \rightarrow 0
\end{aligned}
$$

again by the claim. Therefore, we only have to prove the claim.

Assume $\frac{t_{\lambda}^{2}}{\lambda} \rightarrow a_{0}$ as $\lambda \rightarrow \infty$ with $a_{0} \in(0, \infty]$. By Lemma 3.5

$$
b\left(w_{\lambda}, q, t_{\lambda}\right) \rightarrow a_{0}
$$

which implies that

$$
J_{\lambda}\left(\underline{u}_{\lambda}\right) \rightarrow-\log (\max K)+f\left(a_{0}\right)>-\log (\max K)-1+2 \log 2,
$$

a contradiction. This completes the proof of the Theorem. 


\section{Proof of Theorem 2.4.}

We first consider a simple case

Proposition 4.1. If $P_{+}=-P_{-}$, then for large $\lambda>0$, there exists a solution $v_{\lambda}$ of (3.2) with $v_{\lambda}(x)=v_{\lambda}(-x)\left(\forall x \in S^{2}\right)$ such that $v_{\lambda}-f e^{v_{\lambda}}$ converges to $u_{0} \in H^{1,2}(\Sigma)$ strongly for $\lambda \rightarrow \infty$, where $u_{0}$ is the solution of (3.7) obtained by Moser [M2].

Proof. The proof of the existence of a solution is very similar to the one in [M2 (see also [T]). If $P_{+}=-P_{-}$, Lemma 3.1 says that $K(x)=K(-x)$ for each $x \in S^{2}$. Therefore, we consider a special subspace $H^{s}=\left\{u \in H^{1,2} \mid u(x)=u(-x), \forall x \in\right.$ $\left.S^{2}\right\}$. For each $u \in H^{s}$, there is the improved Moser inequality

$$
\log f_{S^{2}} e^{u} \leq \frac{1}{8} f_{S^{2}}|\nabla u|^{2}+c f_{S^{2}} u
$$

for some constant $c>0$. From this inequality, it is easy to show that $J_{\lambda}$ satisfies the Palais-Smale condition and the coercivity in $H^{s} \cap \mathcal{A}_{\lambda}$. The latter is

$$
J_{\lambda}(u) \geq c_{1} f|\nabla u|^{2}-c_{2} \quad \text { for } u \in H^{s} \cap \mathcal{A}_{\lambda}
$$

for some positive constant $c_{1}, c_{2}$. Actually, by (4.1) one can choose $c=1 / 8$.

Set $\alpha_{\lambda}^{s}=\inf _{u \in H^{s} \cap \mathcal{A}_{\lambda}} J_{\lambda}(u)$. As in section 3, we have

$$
\lim _{\lambda \rightarrow \infty} \alpha_{\lambda}^{s}=\alpha_{0}^{s}-1+2 \log 2
$$

and

$$
\inf _{u \in \partial \mathcal{A}_{\lambda} \cap H^{s}} J_{\lambda}(u) \geq \alpha_{0}^{s}-2+2 \log 4,
$$

where $\alpha_{0}^{s}=\inf _{u \in H^{s}} J(u)$ was studied by Moser in [M2]. By a standard method, we show that $\alpha_{\lambda}^{s}$ is achieved by $u_{\lambda}^{s} \in \mathcal{A}_{\lambda} \cap H^{s}$. The "symmetric variational principle" $[\mathrm{P}]$ implies that $u_{\lambda}^{s}$ is a critical point of $J_{\lambda}$ in $\stackrel{\circ}{\mathcal{A}}_{\lambda}$. Hence $v_{\lambda}=u_{\lambda}^{s}+\rho\left(u_{\lambda}^{s}\right)$ is a solution of (3.2) by Lemma 3.2.

Moreover, (4.2) and (4.3) imply that

$$
f\left|\nabla u_{\lambda}^{s}\right|^{2} \leq c
$$


for some constant $c$, provided that $\lambda$ is large enough. In view of the normalization $f e^{u}=1$. This implies that $u_{\lambda}^{s}$ is bounded in $H^{1,2}$.

Assume $u_{\lambda}^{s} \rightarrow u_{*}^{s} \in H^{1,2}$ weakly in $H^{1,2}$ and strongly in $L^{p}\left(S^{2}\right)$ for any $1<$ $p<+\infty$. As in section 3 , we have $f K e^{u_{\lambda}^{s}} \rightarrow f K e^{u_{*}^{s}}$ and $f K^{2} e^{2 u_{\lambda}^{s}} \rightarrow f K^{2} e^{2 u_{*}^{s}}$. Thus (4.3) implies that $u_{\lambda}^{s}$ converges to $u_{*}^{s}$ strongly in $H^{1,2}$. Clearly, $u_{*}^{s}$ satisfies (3.7) and was obtained in [M2].

Proposition 4.2. If $P_{+}=P_{-}$, there is no solution $v_{\lambda}$ of (3.2) for large $\lambda$ such that $v_{\lambda}-f e^{v_{\lambda}}$ converges strongly in $H^{1,2}$.

Proof. Assume that $u_{\lambda}=v_{\lambda}-f e^{v_{\lambda}}$ converges to $\tilde{u}$ strongly in $H^{1,2}$. It is easy to check that $\tilde{u}$ satisfies (3.7). However, in this case, i.e. $P_{+}=P_{-}$the equation (3.7) admits no solution by the Kazdan-Warner identity

$$
\int\left\langle\nabla K, \nabla x_{i}\right\rangle e^{u}=0
$$

that has to hold for any solution of (3.7), see [KW].

Now we consider the general and more difficult case $P_{+} \neq \pm P_{-}$. In this case, by Lemma 3.1, we know that $K$ has a unique saddle point $Q$ and a unique maximum point $\bar{Q}(=-Q)$. Moreover $\min _{x \in \Gamma} K(x)=K(Q)$, where $\Gamma$ is the great circle crossing $Q$ and $(\bar{Q})$. This $\Gamma$ satisfies the condition (5.1) in [CY2], hence we can define a minimax value of $J_{\lambda}$ as in [CY2] (see also [CkL] and [CD]).

Let $\gamma: \partial D \rightarrow \Gamma$ be a parametrization of $\Gamma$, where $D$ is the unit disc in $\mathbb{R}^{2}$ with boundary $\partial D$.

Definition 4.3. ([CY2]) $\mathcal{D}(\Gamma)=\{h: D \rightarrow H$ is a continuous map with the following asymptotic behavior for all $z_{0} \in \partial D$ :

$$
\begin{gathered}
\lim _{z \rightarrow z_{0}} S(h(z))=0 \\
\left.\lim _{z \rightarrow z_{0}} P(h(z))=\gamma\left(z_{0}\right) \in S^{2}\right\} .
\end{gathered}
$$

(Here, $P$ is the center of mass defined in section 3, and $\left.S(h)=f \frac{1}{2}\left(|\nabla h|^{2}+2 h\right)\right)$.

Set $\beta_{0}=\inf _{h \in \mathcal{D}} \max _{z \in D} J(h(z))$.

Lemma 4.4. ([CY2], $[\mathrm{CkL}]) \beta_{0}>-\log K(Q)+c_{0}$ for a constant $c_{0}>0$.

For our problem, we need to modify the definition of $\mathcal{D}$.

Definition 4.5. $\mathcal{D}^{\prime}=\{h: D \rightarrow H$ is a continuous map satisfying the following 
asymptotic conditions for all $z_{0} \in \partial D$

$$
\begin{gathered}
\lim _{z \rightarrow z_{0}} S(h(z)) \leq \delta \\
\lim _{z \rightarrow z_{0}} P(h(z)) \in B_{\delta}\left(\gamma\left(z_{0}\right)\right)
\end{gathered}
$$

for a fixed small $\delta>0\}$.

We show that for small $\delta, \mathcal{D}^{\prime}$ and $\mathcal{D}$ are essentially the same in the following Lemma.

Lemma 4.6. There exists $\delta_{0}>0$ such that for any $\delta<\delta_{0}$,

(i) $\max _{z \in D} J(h(z))$ is achieved in the interior of $D$ for any $h \in \mathcal{D}^{\prime}$,

(ii) $\beta_{0}^{\prime}:=\inf _{h \in \mathcal{D}^{\prime}} \max _{z \in D} J(h(t))=\beta_{0}$.

Proof. For each $h \in \mathcal{D}^{\prime}$, we first construct an $\tilde{h} \in \mathcal{D}$ such that

(1) $S(\tilde{h}(t)) \leq \delta$ and $P(\tilde{h}(t)) \in B_{\delta}\left(\gamma\left(\frac{z}{|z|}\right)\right)$, if $1 / 2 \leq|z| \leq 1$

(2) $\tilde{h}(z)=h(2 z)$, if $|z| \leq 1 / 2$.

As in section 3 , we decompose $h(z)$ as $\left(w_{z}, t_{z}, q_{z}\right)$ for $z \in D$, where $q_{z}=$ $\frac{P(h(z))}{|P(h(z))|}, 1-t_{z}^{-2} \log t_{z}=|P(h(z))|$ and $w_{z}=h(z) \circ \varphi_{q_{z}, t_{z}}+\psi_{q_{z}, t_{z}}$. By the definition of $\mathcal{D}^{\prime}, q\left(z_{0}\right) \in B_{\delta}\left(\gamma\left(z_{0}\right)\right)$ and $S\left(w_{z}\right)=S(h(z))<\delta$ for any $z_{0} \in \partial D$. We extend $h$ to $D_{2}=\{z \in \mathbb{C}|| z \mid \leq 2\}$ by

$$
h^{\prime}(z)= \begin{cases}\left(w_{z}, q_{z}, t_{z}\right) & \text { if }|z| \leq 1, \\ \left((2-|z|) w_{\mid z}, \tilde{q_{z}}, \tilde{t_{z}}\right) \leq|z| \leq 2, & \end{cases}
$$

where $\tilde{q_{z}}$ and $\tilde{t_{z}}(1 \leq|z| \leq 2)$ are defined by

$$
\tilde{q_{z}}=\frac{Q_{z}}{\left|Q_{z}\right|} \quad \text { and } \quad 1-{\tilde{t_{z}}}^{-2} \log \tilde{t_{z}}=Q_{z}
$$

and

$$
Q_{z}=(2-|z|) P\left(h\left(\frac{z}{|z|}\right)\right)+(|z|-1) \gamma_{\frac{z}{|z|}} .
$$

Since $P\left(h\left(\frac{z}{|z|}\right)\right) \in B_{\delta}\left(\gamma\left(\frac{z}{|z|}\right)\right), h^{\prime}$ is well-defined. Now, let $\tilde{h}(z)=h^{\prime}(2 z)$ for $z \in D$. Clearly, $\tilde{h} \in \mathcal{D}$. By Lemma 4.4. we have

$$
\max _{z \in D} J(\tilde{h}(z)) \geq \beta_{0}>-\log K(Q)+c_{0} .
$$

On the other hand, it is clear that for small $\delta>0 S(u) \leq \delta$ and $P(u) \in B_{\delta}\left(\gamma\left(z_{0}\right)\right)$ for some $z_{0} \in \partial D$ imply that

$$
J(u) \leq-\log K(Q)+\varepsilon_{0}
$$


for $\varepsilon_{0}<c_{0} / 2$. Hence, we have $\max _{z \in D} J(\tilde{h}(z))=\max _{z \in D} J(h(z))$. Now it is clear that (i) and (ii) follow.

Now we return to consider our functional $J_{\lambda}$. Let $\mathcal{D}_{\lambda}=\left\{h \in \mathcal{D}^{\prime} \mid h(D) \subset \mathcal{B}_{\lambda}\right\}$. Recall that $\mathcal{B}_{\lambda}=\{u \in H \mid b(u) \leq \log \lambda\}$. For a fixed $\delta_{0}$ (for example $\delta_{0}$ as in Lemma 4.6) it is clear that $\mathcal{D}_{\lambda} \not \equiv \emptyset$, if $\lambda$ is sufficiently large. Set

$$
\beta_{\lambda}=\inf _{h \in \mathcal{D}_{\lambda}} \max _{z \in D} J_{\lambda}(h(z)) .
$$

Lemma 4.7. $\lim _{\lambda \rightarrow \infty} \beta_{\lambda}=\beta_{0}-1+2 \log 2$.

Proof. In view of Lemma 4.6, for any $\varepsilon>0$ there exists $h_{0} \in \mathcal{D}^{\prime}$ such that

$$
\left|\max _{z \in D} J\left(h_{0}(z)\right)-\beta_{0}\right|<\varepsilon / 2 .
$$

Since $\mathcal{B}_{\lambda} \rightarrow H$ as $\lambda \rightarrow+\infty$, we can choose $\lambda_{0}>0$ such that $h_{0}(D) \subset \mathcal{B}_{\lambda}$ for any $\lambda>\lambda_{0}$. Hence

$$
\begin{aligned}
\max _{z \in D} J_{\lambda}\left(h_{0}(z)\right) & \leq \max J\left(h_{0}(z)\right)+f\left(\frac{1}{1+\sqrt{1-\frac{8}{\lambda} \log \lambda}}\right) \\
& \leq \beta_{0}+\varepsilon-1+2 \log 2
\end{aligned}
$$

provided that $\lambda_{0}$ is large enough. On the other hand, for each $h \in \mathcal{D}_{\lambda}$, we have

$$
\max _{z \in D} J(h(t)) \geq \beta_{0}-1+2 \log 2 .
$$

This proves the Lemma.

Lemma 4.8. $J_{\lambda}$ satisfies the Palais-Smale condition in $\mathcal{A}_{\lambda}$.

In fact, this was proved in the argument of Proposition 3.7.

Now we state our main result in this section.

Proposition 4.9. $\beta_{\lambda}$ is achieved by $\bar{u}_{\lambda} \in \stackrel{\circ}{\mathcal{B}}_{\lambda}$, provided that $\lambda$ is large enough.

Proof. We divide the proof into several steps.

Step 1. We have

(i) For $\varepsilon_{0}>0$ and large $T_{1}$, there exist $M_{0}>0$ and $\gamma_{0}>0$ such that any $u=(w, q, t)$ with $J_{\lambda}(u) \leq \beta_{0}-1+2 \log 2+\varepsilon_{0}$ and $t \geq T_{1}$ satisfies that $S(u)<M_{0}$ and $u=(w, q, t)$ with $q \notin B_{\gamma_{0}}\left(P_{ \pm}\right)$. 
(ii) There exist small $\delta>0$ and large $T_{2}>0$ such that, if $u=(w, q, t)$ with $S(u) \leq \delta, q \in B_{\delta}(\gamma)$ and $t \geq T_{2}$, then $J_{\lambda}(u) \leq \beta_{0}-1+2 \log 2-c_{0} / 2$.

Note that (ii) was used in the proof of Lemma 4.6.

Step 2. From [CkL, p51] there exist $b_{0}, e_{0}, e_{1}, T_{3}$ and $N_{0}, N_{1}>0$ such that if $u=(w, q, t)$ with $q \notin B_{\gamma_{0}}\left(P_{ \pm}\right)$and $S(w) \leq M_{0}$, then

(i) $\left\|\partial_{w} J\right\| \geq e_{0}-N_{0} t^{-1} \log ^{1 / 2} t$, if $S(w) \geq b_{0}$ and $t>T_{3}$,

(ii) $\left\langle\partial_{w} J, \tilde{v}\right\rangle \geq e_{1} S^{1 / 2}(w)-N_{1} t^{-1} \log ^{1 / 2} t$, if $S(w) \leq b_{0}$ and for any $\tilde{v} \in T_{w} H_{0}$.

Here $\partial_{w} J(u)$ is the derivative with respect to $w$. Let $T_{0}=\max \left\{T, T_{1}, T_{2}, T_{3}\right\}$, where $T$ is determined in Lemma 3.5.

Note that in steps 1 and 2 , all constants are independent of $\lambda$.

Step 3. There exists $\lambda_{0}>0$ such that for any $\lambda>\lambda_{0}$

$$
X:=\left\{u \in(w, q . t) \mid t \leq T_{0}\right\} \subset \mathcal{B} \lambda .
$$

$u \in X$

Step 4. If $u=(w, q, t) \in \mathcal{B}_{\lambda}$ with $S(w) \leq M_{0}, q \notin B_{\gamma_{0}}\left(P_{ \pm}\right)$and $t \geq T_{0}$, then

$$
\begin{gathered}
\left\|\partial_{w} f_{\lambda}(w, q, t)\right\| \leq c_{1} \frac{t}{\lambda} \leq c_{1} t e^{-c_{2} t} \\
\left\|\partial_{t} f_{\lambda}(w, q, t)\right\| \leq c_{1} t e^{-c_{2} t^{2}}
\end{gathered}
$$

and

$$
\left\|\partial_{q} f_{\lambda}(w, q, t)\right\| \leq c_{1} \frac{t}{\lambda} \leq c_{1} t e^{-c_{2} t^{2}}
$$

for some positive constant $c_{1}$ and $c_{2}$.

It is enough to check that there exists a constant $c$ such that

$$
\left|\left\langle\partial_{w} d(u), v\right\rangle\right| \leq c t\|v\|
$$

for any $v \in T_{w} H_{0}$.

$$
\begin{aligned}
\left\langle\partial_{w} d(u) v\right\rangle & =\frac{2 \int K^{2} \circ \varphi_{q, t} e^{2 w} \cdot v\left(\operatorname{det}\left(d \varphi_{q, t}\right)^{-1}\right)}{\left(\int K \circ \varphi_{q, t} e^{w}\right)^{2}} \\
& -\frac{2 \int K^{2} \circ \varphi_{q, t} e^{2 w}\left(\operatorname{det}\left(d \varphi_{q, t}\right)^{-1}\right) \int K \circ \varphi_{q, t} e^{w} \cdot v}{\left(\int K \circ \varphi_{q, t} e^{w}\right)^{3}} \\
& \leq \frac{2\left\{K^{4} \circ \varphi_{q, t} e^{4 w}\left(\operatorname{det}\left(d \varphi_{q, t}\right)^{-2}\right\}^{1 / 2}\left(\int|v|^{2}\right)^{1 / 2}\right.}{\left(\int K \circ \varphi_{q, t} e^{w}\right)^{2}} \\
& +\frac{\int K^{2} \circ \varphi_{q, t} e^{2 w}\left(\operatorname{det}\left(d \varphi_{g, t}\right)^{-1}\right)\left(\int K^{2} \circ \varphi_{q, t} e^{2 w}\right)^{1 / 2}\left(\int|v|^{2}\right)}{\left(\int K \circ \varphi_{q, t} e^{w}\right)^{2}}
\end{aligned}
$$




$$
\leq c t^{2}\left(\int|v|^{2}\right)^{1 / 2} \text {, by Lemma } 3.5 \text {. }
$$

Since $u \in \mathcal{B}_{\lambda}$, we have, by Lemma 3.5

$$
c t^{2} \leq \log \lambda .
$$

This is equivalent to $\lambda^{-1} \leq e^{-c t^{2}}$. Step 4 follows.

Step 5. Extend $J_{\lambda \mid X}$ to $Y:=\left\{t \leq T_{0}\right\} \cup\left\{u \in(w, q, t) \mid S(w) \leq M_{0} q \notin B_{\gamma_{0}}\left(P_{ \pm}\right)\right\}$. Let $u \in Y$ define a functional $H: Y \rightarrow \mathbb{R}$ as in [CkL] by

$$
H(w, q, t)=S(w)-\log K(q)-\frac{2 \Delta K(q)}{K(q)} t^{-2} \log t .
$$

One can check that

$$
|J-H| \leq \begin{cases}N_{0} t^{-1} \log ^{1 / 2} t & \text { if } S(w) \geq b t>T_{0} \\ N_{1}\left(|\nabla K(q)| t^{-1} \log ^{1 / 2} t S(w)\right. & \text { if } S(w) \leq b t>T_{0} \\ \left.+t^{-2}+S^{2}(w)\right) & \end{cases}
$$

see [CkL, p51]. Extending $J$ as in [CkL], we obtain $\tilde{J}$ in $Y$. We define $f_{\lambda \mid X}$ smoothly to $\tilde{f}_{\lambda}=Y \rightarrow \mathbb{R}$ such that

(i) $\tilde{f}_{\lambda \mid X}=f_{\lambda \mid X}$,

(ii) $\tilde{f}$ satisfies Step 4 ,

(iii) $\tilde{f}_{\lambda}=-1+2 \log 2$, when $t$ is sufficient large.

Now we obtain a new functional $\tilde{J}_{\lambda}=\tilde{J}+\tilde{f}_{\lambda}$ defined in $Y$ satisfying

(a) $\partial \tilde{J}_{\lambda}(w) \not \equiv 0$ if $u=(w, q, t)$ with $t \geq T_{0}$

(b) $\tilde{J}_{\lambda}$ satisfies the Palais-Smale conditions on $\left(\beta_{\lambda}-\varepsilon_{0}, \beta_{\lambda}+\varepsilon_{0}\right)$ for a fixed small constant $\varepsilon_{0}>0$

(c) $\tilde{J}_{\lambda \mid X}=J_{\lambda \mid X}$

(d) $\tilde{J}_{\lambda}$ satisfies Steps 1-4.

(a) We can follow [CkL] to prove (a). Here we give a sketch.

If $S(w) \geq t^{-1} \log t$, then $\left\|\partial_{w} \tilde{J}\right\| \geq N_{0} t^{-1} \log ^{1 / 2} t$ (see [CkL]). (ii) and Step 4 implies that if $t \geq T_{0}$

$$
\left\|\partial_{w} \tilde{f}\right\| 2 \leq c t e^{-c t^{2}} .
$$

Hence $\left\|\partial_{w} \tilde{J}_{\lambda}\right\| \geq \frac{N_{0}}{2} t^{-1} \log ^{1 / 2} t$, provided that $T_{0}$ is sufficient large.

If $S(w) \leq t^{-1} \log t$, it was shown in [CkL] that near $Q$ or $\bar{Q}$

$$
\left|\partial_{t} \tilde{J}(u)-\frac{2 \Delta K(q)}{K(q)}\right|
$$


is controlled by $c \log ^{-1} t$ if $t \geq T_{0}$ and away from $Q$ and $\bar{Q}$

$$
\left|\partial_{q} \tilde{J}(u)+\frac{\partial_{q} K(q)}{K(q)}\right|
$$

is controlled by $c t^{-1+\mu} \log t$, if $t \geq T_{0}$. Here $0<\mu<1$. By (ii) and Step 4 it is clear that (4.10) and (4.11) hold also for $\tilde{J}_{\lambda}$. Thus there are no critical points of $\tilde{J}_{\lambda}$ if $t \geq T_{0}$.

(b) If $\left\{u_{i}\right\} \subset Y$ is a Palais-Smale sequence for $\tilde{J}_{\lambda}$, the argument in (i) implies that we may assume that $\left\{u_{i}\right\} \subset X$. Hence (b) follows from Lemma 4.8.

(c) and (d) are clear.

Step 6. Now we set $\beta_{\lambda}^{\star}=\inf _{h \in \mathcal{D}_{\lambda}^{\star}} \sup _{z \in D} \tilde{J}_{\lambda}(h(z))$, where $\mathcal{D}_{\lambda}^{\star}=\left\{h \in \mathcal{D}^{\prime} \mid h(D) \subset\right.$ $Y\}$. Using the above argument, we have

$$
\mathcal{D}_{\lambda}^{\star} \not \equiv \emptyset
$$

and

$$
\lim _{\lambda \rightarrow \infty} \beta_{\lambda}^{\star}=\beta_{0}-1+2 \log 2 .
$$

Thus, for large $\lambda>0, \beta_{\lambda}^{\star} \in\left(\beta_{0}-1+2 \log 2-\varepsilon_{0}, \beta-1+2 \log 2+\varepsilon_{0}\right)$. Since $\tilde{J}_{\lambda}$ satisfies the Palais-Smale condition on $\left(\beta_{0}-1+2 \log 2-\varepsilon_{0}, \beta-1+2 \log 2+\varepsilon_{0}\right)$, if $\beta_{\lambda}^{\star}$ is not a critical value of $\tilde{J}_{\lambda}$, we can find a deformation $T(\cdot, t): Y \times[0,1] \rightarrow Y$ $\varepsilon_{1}>0$ such that

(i) $T(u, 0)=u$,

(ii) $T(u, 1) \subset \tilde{J}_{\beta_{\lambda}^{\star}-\varepsilon_{1}}$, if $u \in \tilde{J}_{\beta_{\lambda}^{\star}+\varepsilon_{1}}$

(iii) $T(u, t)=u$, if $u \in \tilde{J}_{\beta_{\lambda}^{\star}-2 \varepsilon_{1}} \cup\left(Y \backslash \tilde{J}_{\beta_{\lambda}^{\star}+2 \varepsilon_{1}}\right)$,

where $\tilde{J}_{b}:=\left\{u \in Y \mid \tilde{J}_{\lambda}(u) \leq b\right\}$ and $\epsilon_{1}<\epsilon_{0} / 4$. The construction of such a deformation is standard. We refer to $[\mathrm{Ck}]$. We claim

$$
T(u, t)=u
$$

if $u \in \partial Y \cup\left\{u=(w, q, t) \mid S(w) \leq \delta, P(u) \in B_{\delta}(\gamma)\right\}$.

If $u=(w, q, t)$ with $S(w) \leq \delta$ and $P(u) \in B_{\delta}(\gamma)$, then by Step $1 J_{\lambda}(u) \leq$ $\beta_{0}-1+2 \log 2-\frac{c_{0}}{2}$. By (iii) in the construction of the deformation $T, T(u, t)=u$. If $u \in \partial Y$, then $u=(w, q, t)$ satisfies either $S(w)=M_{0}, q \in \partial B_{\gamma_{0}}\left(P_{ \pm}\right)$and $t \geq T_{0}$ or $S \geq M_{0}, q \in B_{\gamma_{0}}\left(P_{ \pm}\right)$and $t=T_{0}$. Again by Step 1, we have $J_{\lambda}(u)>$ $\beta_{0}-1+2 \log 2+\epsilon_{0}$, hence $T(u, t)=u$ by (iii) above.

Hence $T \circ h \in \mathcal{D}_{\lambda}^{\star}$ for any $h \in \mathcal{D}_{\lambda}^{\star}$. Now it is clear that the existence of such a deformation contradicts the definition of $\beta_{\lambda}^{\star}$. Therefore $\beta_{\lambda}^{\star}$ is a critical value and there is $\bar{u}_{\lambda} \in Y$ such that $\bar{u}_{\lambda}$ is a critical point of $\tilde{J}_{\lambda}$ and $\tilde{J}_{\lambda}\left(\bar{u}_{\lambda}\right)=\beta_{\lambda}^{\star}$. By the 
construction of $\tilde{J}_{\lambda}$, we know $\bar{u}_{\lambda} \in X$ and $\tilde{J}_{\lambda}\left(\bar{u}_{\lambda}\right)=J_{\lambda}(u)=\beta_{\lambda}$. This completes the proof of the proposition.

Proposition 4.10. By taking a subsequence, $\bar{u}_{\lambda}$ converges to $\bar{u}_{0}$ strongly in $C^{q}$ for any $q>1$, where $\bar{u}_{0}$ is a solution of (3.7) obtained in [CkL] and [CY].

Proof. From the argument in the proof of the previous proposition, we have $\bar{u}_{\lambda} \in X$. Therefore $\bar{u}_{\lambda}$ is bounded in $H^{1,2}$. Assume $\bar{u}_{\lambda}$ converges to $\bar{u}_{0}$ weakly in $H^{1,2}$, strongly in $L^{p}$ for $p>1$ and almost everywhere. As before, by Lebesgue's theorem, we have

$$
\int K e^{\bar{u}_{\lambda}} \rightarrow \int K e^{\bar{u}_{0}} \text { and } \int K^{2} e^{2 \bar{u}_{\lambda}} \rightarrow \int K^{2} e^{2 \bar{u}_{0}}
$$

Hence,

$$
\lambda e^{\rho\left(\bar{u}_{\lambda}\right)} \rightarrow \frac{2}{f K e^{\bar{u}_{0}}}
$$

as $\lambda \rightarrow+\infty$

Since $\bar{v}_{\lambda}=\bar{u}_{\lambda}+\rho\left(\bar{u}_{\lambda}\right)$ satisfies (3.2), i.e.

$$
\int\left\langle\nabla \bar{v}_{\lambda} \cdot \nabla \varphi\right\rangle+\lambda \int K e^{\bar{v}_{\lambda}}\left(K e^{\bar{v}_{\lambda}}-1\right) \varphi+2 \int \varphi=0
$$

we have

$$
\int \nabla \bar{u}_{0} \cdot \nabla \varphi-2 \int K e^{\bar{u}_{o}} \varphi+2 \int \varphi=0,
$$

which implies that $\bar{u}_{0}$ is a solution of (3.7). Choosing $\varphi=u_{\lambda}-u_{0}$ in (4.12) and (4.13), we conclude

$$
\begin{aligned}
\int\left|\nabla \bar{u}_{\lambda}-\nabla \bar{u}_{0}\right|^{2} & =-\lambda \int K e^{\rho\left(\bar{u}_{\lambda}\right)} e^{\bar{u}_{\lambda}}\left(K e^{\rho\left(\bar{u}_{\lambda}\right)} e^{\bar{u}_{\lambda}}-1\right)\left(u_{\lambda}-u_{0}\right) \\
& +2 \int K e^{\bar{u}_{0}}\left(u_{\lambda}-u_{o}\right) \\
& =-\lambda \int K e^{\rho\left(\bar{u}_{\lambda}\right)} e^{\bar{u}_{\lambda}}\left(u_{\lambda}-u_{0}\right) \\
& +2 \int K e^{\bar{u}_{0}}\left(u_{\lambda}-u_{o}\right)+o(1) \\
& =o(1) \quad \text { as } \lambda \rightarrow+\infty
\end{aligned}
$$

It follows that $\bar{u}_{\lambda}$ converges to $\bar{u}_{0}$ in $H^{1,2}$. Now it is easy to conclude that $\bar{u}_{\lambda} \rightarrow \bar{u}_{0}$ in $C^{q}\left(S^{2}\right)$ for any $q \geq 1$ by the elliptic estimates.

Proof of Theorem 2.4. It follows from Proposition 4.9 and 4.10.

Remark. $F_{A_{k}^{2}} \rightarrow 4 \pi \frac{e^{u_{0}+\bar{u}_{0}}}{\int e^{u_{0}+\bar{u}_{0}}}$ as $k \rightarrow 0$, where $u_{0}$ is a solution of $(3.2)$ and $\bar{u}_{0}$ is a solution of (3.7) with $K=e^{u_{0}}$. 


\section{Acknowledgement}

This paper was written at the Max-Planck-Institute for Mathematics in the Sciences in Leipzig. W.Y.D thanks the Max-Planck-Institute for its hospitality and good working conditions. J.Y.L. was supported by a fellowship of the Humboldt Foundation. G.F.W. was supported by the DFG through the Leibniz award of J.J.

\section{References}

[A] T. Aubin, Meilleures constantes dans le theoreme d'inclusion de Sobolev et un theoreme de Fredholm non lineaire pour la transformation conforme de courbure scalaire, J. Funct. Anal. 32 (1979), 148-174.

[CaY] L. Caffarelli and Y. S. Yang, Vortex condensation in the Chern-Simons Higgs model: an existence theorem, Comm. Math. Phys. 168 (1995), 321-336.

[CY1] A. S. Y. Chang and P. Yang, Conformal deformation of metrics on $S^{2}$, J. Diff. Geom. 23 (1988), 259-296.

[CY2] A. S. Y. Chang and P. Yang, Prescribing Gaussian curvature on $S^{2}$, Acta Math. 159 (1987), 214-259.

[Ck] K. C. Chang, Infinite dimensional Morse theory and multiple solution problems, Birkhäuser, 1993

[CkL] K. C. Chang and J. Q. Liu, On Nirenberg's problem, International J. Math. 4 (1993), $35-57$.

[CD] W. X. Chen and W. Y. Ding, Scalar curvature on $S^{2}$, Trans. Amer. Math. Soc. 303 (1987), 365-382.

[DJLW] W. Ding, J. Jost, J. Li and G. Wang, An analysis of the two-vortex case in the ChernSimons Higgs model, Calc. Var. 7 (1998), 87-97.

[H] C. W. Hong, A best constant and the Gaussian curvature, Proc. Amer. Math. Soc. 97 (1986), 737-747.

[HJS] M. C. Hong, J. Jost and Struwe, Asymptotic limits of a Ginzburg-landau type functional, In: J. Jost (Ed.), Geometric Analysis and the Calculus of Variations for Stefan Hildebrandt, Intern. Press Boston, 1996.

[HKP] J. Hong, Y. Kim and P. Y. Pac, Multivortex solutions of the Abelian Chern-Simons theory, Phys. Rev. Lett. 64 (1990), 2230-2233.

[JW] R. Jackiw and E. Weinberg, Self-dual Chern-Simons vortices, Phys. Rev. Lett. 64 (1990), 2234-2237.

[KW] J. Kazdan and F. Warner, Curvature functions for compact 2-manifolds, Ann. Math. 99 (1974), 14-47.

[M1] J. Moser, A sharp form of an inequality of N. Trudinger, Indiana Univ. Math. J. 20 (1971), 1077-1092.

[M2] J. Moser, On a nonlinear problem in differential geometry, In: M. Peixoto (Ed), Dynamical Systems, Academic Press, 1969, pp. 273-280.

[NT] M. Nolasco and G. Tarantello, On a sharp Sobolev type inequality on two dimensional compact manifolds, preprint.

[P] R. Palais, The principle of symmetric criticality, Comm. Math. Phys. 69 (1979), 19-30.

[T] G. Tarantello, Multiple condensate solutions for the Chern-Simons-Higgs theory, J. Math. Phys. 37 (1996), 3769-3796.

[T1] C. H. Taubes, Arbitrary N-vortex solutions to the first order Ginzburg-Landau equations, Commun. Math. Phys. 72 (1980), 277-292.

[T2] C. H. Taubes, On the equivalence of the first and second order equations for gauge, 
Commun. Math. Phys. 75 (1980), 207-227.

[T3] C. H. Taubes, The Seiberg-Witten invariants and Gromov invariants, Math. Res. Lett. 2 (1995), 221-238.

Weiyue Ding, Jiayu Li, Guofang Wang Institute of Mathematics

Academia Sinica, Beijing 100080

P. R. China

(Received: December 3, 1997)
Jürgen Jost

Max-Planck-Institute

for Mathematics in the Sciences,

Inselstr. 22-26

D-04103 Leipzig, Germany 